educated, male, have poorer cognitive performance, and a history of depression. (3) PGD is characterized by sadness, fascination with or excessive avoidance of the memories associated with the deceased, the memory of the loss and mental ruminations about death. According to some investigations, PGD reduces life expectancy, increasing the odds of death, causes severe behavioural symptoms, and is associated with longer-term functional impairment. $(4,5)$

Conclusion: Given these results, it is easy to understand the need for a closer monitoring of these patients during this global crisis. Moreover, it is also important to find new ways to do that while complying with COVID-19 rules and restrictions. A short-term evidence-based intervention for PGD effective in the elderly is based on support focused upon these seven themes: understanding their grief, managing emotional pain, thinkingabout the future, strengthening their relationships, telling the story of the dead, learning to live with reminders of the deceased, and connecting with memories. (3) 1 Goveas, JS, Shear, MK. Grief and the COVID-19 Pandemic in Older Adults. Am J of Geriatric Psychiatry (2020). 28:10,1119-1125. https://doi.org/10.1016/j.jagp.2020.06.021

2 Ishikawa, RZ. I May Never See the Ocean Again: Loss and Grief Among Older Adults During the COVID-19 Pandemic.J. Psychol. Trauma (2020) Vol. 12, No. S1, S85-S86. http://dx.doi.org/10.1037/tra0000695

3 Meichsner, F, O'Connor, M. et al. Grief Before and After Bereavement in the Elderly: An Approach to Care. Am J ofGeriatric Psychiatry (2020). 28:5, 560-569. https://doi.org/10.1016/j.jagp.2019.12.010

4 Prigerson, HG., Frank, E. et al. Complicated Grief and Bereavement-Related Depression as Distinct Disorders:Preliminary Empirical Validation in Elderly Bereaved Spouses. AmJPsychiatry (1995) 152:1

5 Morowatisharifabad, MA., Alizadeh, A. et al. Prevalence of complicated grief and related factors in elderly individuals in Sabzevar City, Iran. Psycogeraitrics (2020). doi:10.1111/psyg.12579

\title{
535 - Loneliness and social isolation in attendees of a Psychiatry of Old Age Service in the North West of Ireland: preliminary findings of a cross-sectional study during the COVID- 19 pandemic.
}

\section{Author List}

Clodagh Rushe, Bernadette Kevin, Ericka Maye, Gavin Sweeney, Kevin McLaughlin, Marguerite Cryan, Aislinn Gannon, Vincent Melvin, Valerie Cogan, Donna Wilkie, Elaine McDonagh, Geraldine McCarthy, Catherine Dolan.

\section{Aims and Hypothesis}

The primary aim is to establish the prevalence of loneliness and social isolation in older adults referred to a Psychiatry of Old-Age Service in the North-West of Ireland. Secondary aims include exploring associations between loneliness, social isolation and, well-being, depression, cognition and healthstatus. The overarching aim was to improve patient outcomes by tailoring mental health and social interventions appropriate to patient need.

\section{Background}

Loneliness is a discrepancy between the social-relations one has and their desired level. It is estimated that one third of older adults will experience loneliness, which along with social isolation has links to poorer health-outcomes, reduced quality of life and cognitive decline. Government advice in Ireland to reduce social activity due to COVID-19 pandemic may compound social disconnection. We present preliminary findings of an ongoing study investigating loneliness, social isolation and related factors in older adults referred to a Mental-Health Service in the North-West of Ireland in 2020 - 2021. 


\section{Methods}

Using a cross-sectional study design, participants completed University of California, Los-Angeles(UCLA) Loneliness Scale (UCLA maximum score $=10$ ); and Berkman-Syme Social Network Index. Quality of life is measured using WHO-Well-being Index(WHO-5) with a number of relevant personal, clinical and social factors also captured.

\section{Results}

Data from 98 questionnaires (January 2020- May 2021), 52\% female, showed average participant age was 74.4 years. Average perceived loneliness score was 3.67 and $85.7 \%,(n=84)$ reported some loneliness (UCLA $>0$ ) with $2 \%(n=2)$ reporting high loneliness levels (UCLA $=10)$. The majority, $77.5 \%$ $(n=76)$ were socially isolated; $35.7 \%(n=35)$ 'mostly isolated', $41.8 \%(n=41)$ 'moderately isolated'. Females were noted to be more isolated.

\section{Conclusions}

Preliminary results illustrate majority of older adults referred to a mental-health service over a timeperiod spanning COVID-19 pandemic are lonely and socially isolated. This is likely compounded by changes to daily routines during COVID-19 pandemic. This is concerning given the adverse health implications. We hope final results will guide enhancement of clinical-care through linkage of mentalhealth services with community agencies, social-care supports and e-health technologies.

\section{6 - Moderating effect of the COVID-19 pandemic on the efficacy of cognitive stimulation. A controlled trial.}

Authors:

Gurbindo-Elizari, Mạ Concepción ${ }^{1}$

Martínez-Martínez, Blanca ${ }^{2}$

Garcia-Casal, J. Antonio ${ }^{3}$

Gómez-Gil, Fernando ${ }^{1}$

${ }^{1}$ Nurse, Servicio Navarro de Salud, Pamplona, Spain.

${ }^{2}$ Occupational Therapist, Servicio Navarro de Salud, Pamplona, Spain.

${ }^{3}$ Clinical Psychologist, Servicio Navarro de Salud, IdiSNA, Pamplona, Spain.

Background:

It's been proved that cognitive stimulation (CS) has direct effects over the improvement of general cognitive functions in people with cognitive impairment (PCI). The restrictions in daily life associated to COVID-19 pandemic had an impact in the quality of life of $\mathrm{PCl}$ and it might have affected the efficacy of the CS programs targeting this population.

Research Objective:

To analyse if there was a moderating effect of the pandemic on the efficacy of CS programs.

Method:

Participants were enrolled in a public memory clinic; $213 \mathrm{PCl}$ were assigned to two groups: 173 received CS treatment before the pandemic (PRECOVID) and 40 received CS during the pandemic (COVID). Pre and post assessments were carried out with the Mini Mental State Exam (MMSE), the clock-test and the brief Geriatric Depression Scale. The treatment consisted of 32 sessions of CS held twice a week during 4 months. No significant differences $(p<.05)$ were found between groups at baseline in age $(74.46 \pm 7.80$ years), cognitive function (MMSE=23.43 \pm 3.30 ), gender ( $58 \%$ women) and the remaining variables. 